\section{Response to: 'Regarding microRNA-181a-5p antisense oligonucleotides attenuate osteoarthritis in facet and knee joints' by Liebling}

We wish to thank Dr Liebling for the relevant comments ${ }^{1}$ regarding our recent article in Annals of the Rheumatic Diseases entitled 'MicroRNA-181a-5p antisense oligonucleotides attenuate osteoarthritis in facet and knee joints'. ${ }^{2}$ We are delighted that our article has gathered such interest in the scientific community and are happy to provide additional comments. In the aforementioned publication, we determined the potential of locked nucleic acid, antisense oligonucleotides against microRNA-181a-5p (LNA-miR-181a-5p ASO) as a therapy for the treatment of osteoarthritis (OA). ${ }^{2}$ The chemical modification of LNA is one of the most advanced ASO delivery systems. LNA modifications enable design of oligonucleotides that are short while maintaining high affinity and stable binding towards targets. ${ }^{3}$

Regarding the first comment by Dr Liebling, we did not employ lipofection reagent for in vivo grade LNA-miR-181a$5 \mathrm{p}$-ASO studies, while we use the reagent to transfect in vitro grade LNA-miR-181a-5p ASO into chondrocytes. As Dr Liebling mentioned, the in vivo LNA-miR-181a-5p ASO used in our studies is 16 nucleotides long, 5 nucleotides shorter than the LNA-miR-181a-5p ASO used for in vitro studies (21 nucleotides in length). This difference in length allows the in vivo LNA-miR$181 \mathrm{a}-5 \mathrm{p}$ ASO to be endocytosed more efficiently via natural cellular uptake mechanisms and explains why shorter LNA-ASO are significantly more potent when administered to animals in vivo. ${ }^{4}$ We agree that LNA-ASO can be added directly to cell cultures in vitro without the need for transfection reagents; however, we have not tested if a difference exists in the uptake or efficacy of the oligonucleotides delivered with or without transfection reagent in chondrocyte cultures in in vitro experiments. In addition, we followed the methodology outlined in our previous publication ${ }^{5}$ to accurately compare cellular effects and efficacy of suppression of endogenous miR-181a-5p to this previous work.

Second, as Dr Liebling indicated, we observed increased Annexin $\mathrm{V}+/ 7-\mathrm{AAD}+$ positivity in human knee OA chondrocytes when using LNA-miR-181a-5p ASO at baseline (without interleukin (IL)-1 $\beta$ ) compared with the scramble control oligonucleotides (figure 6D and online supplementary figure 13). This effect is not observed in mouse or rat cells (online supplementary figures 3 and 4). Thus, this human chondrocyte response could be a result of species-selective 'off-target effects' of the in vitro grade LNA-miR-181a-5p ASO, which we concede as a limitation in the discussion section of our published work. ${ }^{2}$ However, LNA-miR-181a-5p ASO is effective at reducing chondrocyte cell death in vitro and in vivo under disease-mimicking conditions (with either IL-1 $\beta$-treatment or surgical induction). In particular, we clearly observe cartilage protective effects of the in vivo grade LNA-miR-181a-5p ASO in both injury-induced facet joint $\mathrm{OA}$ and trauma-induced knee OA animal models.
Nevertheless, as Dr Liebling mentioned, in vitro grade LNAmiR-181a-5p ASO does initiate more human chondrocyte cell death than scramble control oligonucleotides in non-disease conditions in vitro (figure 6D). As it is possible that the in vivo grade LNA-miR-181a-5p ASO has less off-target effects, it will be necessary to study in vivo and in vitro grade LNA-miR-181a-5p ASO in culture studies both with and without IL-1 $\beta$-treatment to compare effects on chondrocyte apoptosis. These potential toxicity-related issues will need to be addressed in future preclinical studies prior to initiating human clinical trials. If any future clinical trials are commenced using this technology, it will be crucial to carefully select and monitor patient participants for any potential adverse events.

Akihiro Nakamura, ${ }^{1,2,3,4}$ Jason S. Rockel, ${ }^{1,2}$ Mohit Kapoor ${ }^{1,2,5,6}$
${ }^{1}$ Arthritis Program, University Health Network, Toronto, Ontario, Canada
${ }^{2}$ Division of Genetics and Development, Krembil Research Institute, University Health
Network, Toronto, Ontario, Canada
${ }^{3}$ Division of Rheumatology, Toronto Western Hospital, University Health Network,
Toronto, Ontario, Canada
${ }^{4}$ Institute of Medical Science, University of Toronto, Toronto, Ontario, Canada
${ }^{5}$ Department of Surgery, University of Toronto, Toronto, Ontario, Canada
${ }^{6}$ Department of Laboratory Medicine and Pathobiology, University of Toronto,
Toronto, Ontario, Canada

Correspondence to Dr. Mohit Kapoor, Arthritis Program, University Health Network, Toronto, Ontario, Canada; mkapoor@uhnresearch.ca

Handling editor Josef S Smolen

Competing interests None declared.

Patient consent for publication Not required.

Provenance and peer review Commissioned; internally peer reviewed.

(c) Author(s) (or their employer(s)) 2020. No commercial re-use. See rights and permissions. Published by BMJ.

\section{Check for updates}

To cite Nakamura A, Rockel JS, Kapoor M. Ann Rheum Dis 2020;79:e70.

Received 13 April 2019

Accepted 14 April 2019

Published Online First 2 May 2019

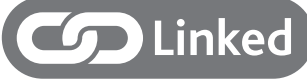

- http://dx.doi.org/10.1136/annrheumdis-2019-215454

Ann Rheum Dis 2020;79:e70. doi:10.1136/annrheumdis-2019-215509

\section{REFERENCES}

1 Liebling M. Regarding micro-RNA-181a-5p antisenseoligonucleotides attenuate osteoarthritis infacet and knee joints. Ann Rheum Dis 2020;79:e70.

2 Nakamura A, Rampersaud YR, Nakamura S, et al. microRNA-181a-5p antisense oligonucleotides attenuate osteoarthritis in facet and knee joints. Ann Rheum Dis 2019;78:111-21.

3 Petersen M, Nielsen CB, Nielsen KE, et al. The conformations of locked nucleic acids (LNA). J Mol Recognit 2000;13:44-53.

4 Straarup EM, Fisker N, Hedtjärn M, et al. Short locked nucleic acid antisense oligonucleotides potently reduce apolipoprotein B mRNA and serum cholesterol in mice and non-human primates. Nucleic Acids Res 2010;38:7100-11.

5 Nakamura A, Rampersaud YR, Sharma A, et al. Identification of microRNA-181a$5 p$ and microRNA-4454 as mediators of facet cartilage degeneration. JCI Insight 2016; 1:e86820. 